\title{
Determinantes das desigualdades na auto-avaliação do estado de saúde no Brasil: análise dos dados da PNAD/1998
}

\author{
Factors determining inequalities in the health \\ condition self-assessment in Brazil: analysis \\ of data of PNAD/1998
}

\footnotetext{
1 Programa de Políticas Públicas e Saúde Divisão de Saúde e Desenvolvimento Humano. Organização Pan-Americana da Saúde. 525, 23rd Street N.W. Washington, D.C. 20037, EUA.

dachsnor@paho.org
}

\begin{abstract}
The work presents a general picture of the inequalities in the determinants considered to explain the disparities in the self-evaluation of health status using the data of the $\mathrm{PNAD} / 1998$. It is shown that there are large gradients in the classification of health status according to levels of education and income per capita, according to race or color of the skin, for the great regions of the country and specially with age. Using logistic regression models an attempt is made to explain which are the most important determinants of this self-classification. The results show that education and income have separate additive effects and that there are differences between men and women and according to living in urban or rural areas. The inequalities in the self-evaluation of health status according to race or color of the skin are no longer statistically significant after controlling for education and income levels. A discussion is presented of the usefulness of this kind of information on self-reported health status and of the importance of improving the quality of this type of data in future household surveys by presenting suggestions on changes in the way the interview is conducted.
\end{abstract}

Key words Self-evaluation of health status, Health inequalities, Household surveys, Determinants of health inequalities
Resumo O trabalho apresenta um panorama geral das desigualdades nos determinantes considerados na explicação das desigualdades na auto-avaliação do estado de saúde a partir dos dados da PNAD/1998. Mostra-se como existem gradientes na classificação do estado de saúde autopercebido de acordo com os níveis de educação, de renda per capita, de acordo com a raça ou cor de pele das pessoas, por grandes regiões do país e especialmente com o aumento da idade. Usando modelos de regressão logística, tenta-se explicar quais as determinações importantes dessa autoclassificação. Os resultados mais importantes indicam que educação e rendimento têm efeitos que se somam e que há diferenças entre homens e mulheres e de acordo com populações urbanas e rurais. As desigualdades na classificação do estado de saúde de acordo com a raça ou cor de pele das pessoas deixam de ser estatisticamente significativas depois de se controlar por nivel de educação e de renda. Discute-se a utilidade desse tipo de informação sobre classificação autopercebida de saúde e a importância de melhorar, em futuros inquéritos, a qualidade dos dados por meio de sugestões sobre alterações nos procedimentos de entrevista.

Palavras-chave Auto-avaliação do estado de saúde, Desigualdades em saúde, Inquéritos de domicílio, Determinantes de desigualdades de saúde 


\section{Introdução}

A região das Américas é, entre todas no mundo, a que apresenta maiores desigualdades internas do ponto de vista de situação socioeconômica de seus grupos populacionais e de condições materiais de vida em geral (IADB, 1999). Isso se reflete em grandes desigualdades em saúde, tanto no estado de saúde de grupos populacionais, como no acesso e utilização de serviços de atenção à saúde (Casas et al., 2001). As reais dimensões dessas desigualdades e das cadeias de determinação das mesmas ainda são pouco conhecidas na América Latina e no Caribe (Almeida Filho, 1998).

Neste trabalho, além de descrever a variável sobre autopercepção relativa ao estado geral de saúde da população brasileira, procura-se estabelecer relações de determinação entre as condições socioeconômicas de indivíduos e grupos familiares e este estado geral de saúde autopercebido, a partir de dados da Pesquisa Nacional por Amostragem de Domicílio (PNAD) de 1998.

Além de ilustrar os enormes graus de desigualdades observados, essas análises permitirão um aprimoramento do desenho dos módulos de saúde de futuros inquéritos nacionais. Os resultados sugerem também algumas alternativas de políticas que poderiam ter um impacto na diminuição de algumas das desigualdades observadas.

Os inquéritos de domicílios de propósitos múltiplos, por meio de entrevistas, são a fonte privilegiada para estudar as relações de determinação de desigualdades em saúde de grupos populacionais (Dachs, 2002; Ravaillon, 1994). Várias são as razões para que assim seja. As mais importantes entre elas são a obtenção, a partir dessa fonte, de informação sobre variáveis de tipo socioeconômico dos grupos familiares e dos indivíduos, simultaneamente com informações sobre estado de saúde, restrições de atividades da vida diária, necessidades percebidas de saúde, acesso e uso de serviços de atenção, bem como de gastos com bens e serviços de atenção à saúde. É relativamente baixo o custo para se obter a informação sobre grandes grupos de população, uma vez que tais inquéritos se realizam periodicamente para coleta de dados sobre as variáveis de emprego, rendimento e situação de moradia. Adicionar um módulo de saúde a esses inquéritos representa em geral um aumento não substancial em tempos de entrevista e geração de arquivos de dados limpos e consistentes.
Outra razão que aumenta a importância dos dados de inquéritos é que os dados de registros de tipo administrativo, em geral, não permitem estudar desigualdades e suas determinações sociais de forma satisfatória, por duas razões. Em primeiro lugar, os registros não incluem informação sobre educação, rendimento, gastos dos domicílios e condições das vivendas. Além de não programados para tal, nem mesmo faria sentido solicitar aos usuários dos serviços que informassem sobre essas dimensões ao buscar atenção. Por outro lado, a cobertura dos sistemas de registro inclui apenas aqueles indivíduos que buscaram atenção, faltando, portanto, os dados sobre os que não recorreram ao sistema ou porque não necessitaram, ou porque não tiveram acesso seja por restrições de tipo geográfica, econômica, cultural, etc. Dessa forma, a partir dos dados de registro, é impossível ir além de uma descrição incompleta e muitas vezes de tipo exclusivamente ecológico, apresentando resultados por áreas geográficas, sem poder estender as análises aos níveis de relações entre variáveis individuais e familiares, além de poder facilmente conduzir a inferências indevidas por problemas metodológicos relacionados com a falácia ecológica.

Por essas razões, uma pesquisa na literatura recente sobre desigualdades em saúde e suas determinações mostra que a quase totalidade dos trabalhos usa dados de inquéritos para a parte empírica dos estudos (Almeida Filho et al., 2002). As exceções encontradas são, na sua maioria, estudos de mortalidade naqueles países em que o sub-registro é muito baixo e que incluem em seus atestados de óbito dados que permitam caracterizar alguma dimensão de natureza socioeconômica do falecido.

Nos módulos de saúde desses inquéritos se incluem perguntas sobre o estado de saúde dos entrevistados, com diferentes graus de profundidade e cobrindo uma ou mais dimensões do estado de saúde. Necessariamente, por tratar-se de inquéritos por entrevista, o que se obtém é informação sobre autopercepção do estado de saúde. Na sessão de materiais, discutem-se as várias perguntas desse tipo existentes na PNAD/ 98, suas características, potenciais problemas e possível qualidade da informação.

Neste trabalho, são descritos os resultados observados para a pergunta sobre estado geral de saúde, e estudadas algumas das possíveis cadeias de determinação do estado de saúde na forma de autopercepção do mesmo, a partir de dados da PNAD/98. Essa pesquisa incluiu um 
módulo sobre as "características de saúde dos moradores" com 79 perguntas que vão desde a pesquisa de autopercepção do estado de saúde, autopercepção de condições e agravos específicos, acesso (incluindo planos e seguros de saúde), utilização de serviços e gasto com bens e serviços de saúde e um módulo sobre "características de mobilidade física dos moradores de 14 anos e mais de idade" com nove perguntas.

\section{Materiais}

Nesta seção são discutidas as perguntas utilizadas na análise. Na PNAD/98 existem dois módulos com perguntas relacionadas com autopercepção do estado de saúde. No módulo 13 sobre "características de saúde dos moradores" as perguntas de números 3 a 20 sobre morbidade são sobre autopercepção do estado geral de saúde, interrupção de atividades por motivo de saúde, e autopercepção de 12 condições ou agravos específicos. Os itens 1 e 2 são também de extrema importância, já que indicam quem foi o informante desta parte da entrevista. No módulo 14 sobre "características de mobilidade física dos moradores de 14 anos e mais de idade" se pergunta sobre 9 tipos de restrições de atividades da vida diária por motivo de saúde. Da mesma forma que no módulo 13, os dois primeiros itens identificam a pessoa que informou sobre esta parte. Somente a pergunta 3 será analisada neste trabalho, acompanhada da identificação de quem a respondeu.

A autopercepção do estado geral de saúde, expressado como "muito bom", "bom", "regular", "ruim" e "muito ruim" é a pergunta a ser analisada, especialmente no que se refere a estudar cadeias de determinação de desigualdades. Os resultados de perguntas como essa estão fortemente relacionados com mortalidade futura e, como se demonstra, associam-se fortemente com o estado "real" ou "objetivo" de saúde das pessoas (Appels et al., 1996; Idler et al., 1990; Leung et al., 1997; McCallum et al., 1994; Sundquist e Johansson, 1997). O principal problema apresentado por esse tipo de pergunta deve-se ao fato de diferentes pessoas e diferentes grupos sociais e étnicos terem diferentes percepções sobre os diferentes níveis que se oferecem como possível resposta (Murray e Chen, 1992; Kroeger, 1983 e 1985; Groot, 2000). Existem também importantes diferenças culturais (Johnson et al., 1996) que determinam que em certos contextos os adultos maiores considerem "natural" o fato de que sua saúde esteja deteriorada (Knäuper et al., 1995; Beckett et al., 2000) e respondam que seu estado de saúde é bom ou muito bom, mesmo em condições de presença de sérios problemas. A comparação entre países torna-se particularmente difícil (Dachs et al., 2002).

Como no trabalho de campo da PNAD/98, por questões de custo, não se visitou o domicílio mais vezes para obter sempre as respostas a esses itens por parte das próprias pessoas (pelo menos para aquelas de 14 ou mais anos de idade). Na realidade, o que se tem muitas vezes não é a classificação de auto-avaliação do estado de saúde, mas a avaliação subjetiva por parte de outra pessoa (o/a entrevistado/a) do estado de saúde dos demais indivíduos (não presentes no domicílio no momento da entrevista).

As características socioeconômicas de indivíduos e dos domicílios que se utilizam para o estudo de relações de determinação das variáveis de estado de saúde são o nível de rendimento do domicílio medido pelo rendimento total per capita, nível de educação dos indivíduos, região geográfica, áreas urbanas e rurais, cor da pele e sexo para capturar diferenças de gênero. Como o estado de saúde varia fortemente com a idade, esta variável foi incluída nos modelos. Todas estas variáveis se obtêm do mesmo conjunto de dados da pesquisa.

\section{Métodos}

Uma das principais variáveis distribucionais a ser utilizada nas análises é a renda per capita dos indivíduos do domicílio. A renda per capita foi calculada dividindo a renda total do domicílio (uma das variáveis adicionadas pelo próprio IBGE ao conjunto de dados) pelo número de pessoas do domicílio.

Existem na literatura econômica grandes debates (Deaton, 1994; Bollen et al., 2001) sobre se em estudos de desigualdade de saúde deve-se utilizar a renda per capita, a renda domiciliar ou uma renda per capita ajustada. A decisão de utilizar neste estudo a renda per capita se deve a dois fatores principais:

- É injusto utilizar a renda domiciliar como variável distributiva quando se deseja estudar as relações entre condições econômicas e saúde, uma vez que os domicílios de rendas elevadas tendem a constituir-se por um número menor de indivíduos. Isso significaria atribuir melhores condições econômicas relativas aos domicí- 
lios em piores níveis de renda simplesmente porque têm mais membros. Com dados da própria PNAD/98 se observa que no decil de menor renda per capita o número médio de pessoas no domicílio é de aproximadamente 6,3, enquanto no decil de maior renda per capita este número é de apenas 3,6.

- Em geral, as propriedades distributivas não mudam apreciavelmente ao se fazer o ajuste por um fator de escala até dois (Valdivia, 2001). Por outro lado, as fórmulas de correção para número de adultos equivalentes são complexas, difíceis de explicar e de entender e até certo ponto bastante arbitrárias. Existe até o argumento de que se deve usar renda por número de adultos do domicílio, especialmente quando se estudam características de saúde de crianças (Montgomery et al., 1999).

É também importante considerar que essa é uma das poucas variáveis com um número relativamente grande de casos de falta de informação. Existem na amostra 2.351 domicílios (aproximadamente $2,5 \%$ depois de expandido) em que não se dispõe do valor da renda. Existem também 1.432 domicílios nos quais a renda total é zero. Este último caso é compreensível. Seria até de se esperar domicílios com renda negativa, se fossem consideradas outras formas de cálculo do mesmo, domicílios que durante o período de referência da pesquisa estão gastando mais do que o total de rendimentos monetários, usando possivelmente recursos poupados anteriormente ou recorrendo a empréstimos.

A variável de estado geral de saúde é descrita segundo sua distribuição por nível de renda per capita do domicílio por decis da distribuição do rendimento. Para esse fim usam-se tabulações simples. O grau de desigualdade existente se expressa tanto como razão de extremos entre o decil de menores rendimentos e o de maiores rendimentos per capita como por curvas e índices de concentração (Dachs, 2001).

Para a variável de estado geral de saúde se criam duas variáveis indicadoras, uma para "estado de saúde muito bom" e outra para "estado de saúde ruim e muito ruim". Desenvolvem-se então modelos de regressão logística para a probabilidade de que uma pessoa seja declarada como tendo cada um desses estados de saúde, usando como variáveis explicativas todas as variáveis de tipo socioeconômico. Nos dois casos as primeiras variáveis que se introduzem no modelo são indicadores de quem fez a classificação do estado de saúde: outro morador do próprio domicílio ou um não morador, consi- derando como referência o caso em que a classificação foi feita pela própria pessoa. A seguir, se incluem indicadores para os vários grupos de idade, considerando o grupo de menores de um ano como referência, seguida da variável "anos de educação", que varia de 0 a 15 , e da variável de renda na forma de decis de renda per capita, variando de 1 a 10. Nos casos em que o número de anos de escolaridade era superior a 15, codificou-se também como 15 após confirmar que praticamente não existem diferenças para 16 anos e mais, além de se tratar de uma porcentagem muito pequena de casos. Em seguida, são introduzidos, nessa ordem, o sexo da pessoa na forma de uma variável indicadora de sexo feminino, um indicador de área rural e indicadores para cor da pele ou raça, tomando como referência cor de pele branca.

Por serem dados de um inquérito com uma amostra complexa, é necessário dar-lhes um tratamento com as técnicas apropriadas para este tipo de estudo (Silva, 2002). Nas partes apenas descritivas é suficiente ter o cuidado de usar os pesos adequados, mas no caso dos modelos de regressão logística deve-se tomar em conta a estrutura da amostragem utilizada. Foi usado o pacote estatístico Stata versão 6 que contém uma série de procedimentos precedidos pela sigla SVY (de survey), considerando os estratos da amostra, e o último nível de aglomeração para caracterizar as unidades primárias de amostragem. No arquivo de dados da PNAD existe a variável UPA para indicar esta última, mas para os estratos foi necessário obter do Departamento de Métodos do IBGE um arquivo especial com duas variáveis, uma que identifica o domicílio e outra que identifica o estrato amostral correspondente, uma vez que a variável "Estrato" existente nos arquivos originais não expressa a divisão em estratos do processo de amostragem.

\section{Resultados}

Apesar de não ser importante para o estudo distribucional que se apresenta, é interessante conhecer quais são os valores em Reais de setembro de 1998 para os quintis e decis de renda per capita. Os decis são os seguintes: primeiro, até 31,43 reais; segundo até 50 ; o terceiro até 72 ; o quarto até 97,$5 ;$ o quinto até 127,$5 ;$ o sexto até 165 ; o sétimo até 223,75 ; o oitavo até 318,67 ; o nono até 550; e o décimo de mais de 550 reais mensais. Os quintis, portanto, são: o primeiro até 50, o segundo até 97,5, o terceiro até 165 , o 
quarto até 318,67 e o quinto de mais de 318,67 reais mensais.

Os decis e quintis de renda per capita foram calculados para o total do país. Uma grande parte das desigualdades regionais que serão observadas se devem indiretamente às grandes diferenças de distribuição da riqueza entre as grandes regiões do país. Na tabela 1 são apresentadas as porcentagens de pessoas nos decis de renda per capita para as cinco regiões. A única que é semelhante ao total do país é a região Centro-Oeste. No Nordeste, mais de 39\% das pessoas estão nos dois primeiros decis e na região Sudeste, mais de $27 \%$ encontram-se no dois decis superiores.

Todas as variáveis consideradas estão relacionadas entre si, na maioria das vezes de forma bastante forte. Numa seqüência de descrições bivariadas apresentam-se algumas das mais importantes.

A raça ou cor da pele estão fortemente associadas aos níveis de renda e de educação. A tabela 2 mostra as porcentagens de pessoas em cada decil de renda per capita de acordo com a

Tabela 1

Porcentagens de pessoas por decis de renda per capita do país, segundo as grandes regiões, Brasil, 1998.

\begin{tabular}{lcccrrr}
\hline $\begin{array}{l}\text { Decis de renda } \\
\text { per capita }\end{array}$ & Norte & Nordeste & Sudeste & Sul & Centro- Oeste & Total \\
\hline 1 & 11,72 & 21,36 & 4,57 & 5,73 & 5,27 & 10,02 \\
2 & 14,47 & 17,98 & 5,62 & 6,74 & 8,34 & 10,00 \\
3 & 13,2 & 14,7 & 7,44 & 7,92 & 10,38 & 10,11 \\
4 & 11,5 & 11,63 & 8,70 & 9,36 & 11,78 & 10,01 \\
5 & 10,62 & 8,59 & 9,91 & 11,02 & 11,68 & 9,86 \\
6 & 9,50 & 7,65 & 11,23 & 11,47 & 11,85 & 10,19 \\
7 & 7,88 & 5,40 & 12,11 & 11,86 & 10,54 & 9,81 \\
8 & 8,17 & 4,45 & 13,17 & 12,31 & 9,48 & 10,00 \\
9 & 7,35 & 4,12 & 13,57 & 12,26 & 9,73 & 10,05 \\
10 & 5,58 & 4,12 & 13,67 & 11,33 & 10,95 & 9,95 \\
Total & 100,00 & 100,00 & 100,00 & 100,00 & 100,00 & 100,00 \\
\hline
\end{tabular}

Fonte: PNAD/1998

$\mathrm{Na}$ coluna de totais, as diferenças em relação a $10 \%$ se devem à existência de muitos

valores iguais para renda e cada limite dos quantis é tomado como um dos valores observados.

Tabela 2

Porcentagens de pessoas em cada decil de renda per capita, de acordo com a sua classificação de raça ou cor da pele, Brasil, 1998.

\begin{tabular}{lrrrrrr}
\hline Decis de renda & \multicolumn{5}{c}{ Cor da pele ou raça } \\
per capita & Indígena & Branca & Preta & Amarela & Parda & Total \\
\hline 1 & 9,2 & 5,45 & 11,59 & 3,23 & 16,12 & 10,02 \\
2 & 11,95 & 6,23 & 12,48 & 1,35 & 14,90 & 10,00 \\
3 & 14,25 & 7,53 & 12,34 & 3,37 & 13,39 & 10,11 \\
4 & 10,24 & 8,33 & 11,75 & 3,08 & 12,13 & 10,01 \\
5 & 10,21 & 9,38 & 10,92 & 2,81 & 10,46 & 9,86 \\
6 & 9,88 & 10,61 & 12,20 & 5,24 & 9,39 & 10,19 \\
7 & 10,19 & 11,16 & 10,77 & 7,50 & 7,86 & 9,81 \\
8 & 9,45 & 12,65 & 7,58 & 8,34 & 6,75 & 10,00 \\
9 & 6,58 & 13,54 & 6,44 & 23,17 & 5,66 & 10,05 \\
10 & 8,08 & 15,13 & 3,94 & 41,91 & 3,33 & 9,95 \\
Total & 100,00 & 100,00 & 100,00 & 100,00 & 100,00 & 100,00 \\
\hline
\end{tabular}

Fonte: PNAD/1998 
sua classificação de raça ou cor da pele. Algumas oscilações para as classificações indígena e amarela se devem, em parte, aos pequenos números de casos na amostra em certas categorias de renda per capita. Apenas cerca de $18 \%$ das pessoas pretas estão nos três decis superiores de renda per capita. Para os de cor parda, essa cifra é ainda menor, cerca de 15,7\%. Já entre brancos, $41,3 \%$ estão nos três decis superiores, e para os amarelos a porcentagem alcança $73,4 \%$. Entre os indígenas a distribuição é mais próxima da uniforme. A figura 1 mostra as curvas de concentração do rendimento per capita de acordo com a raça ou cor da pele.
Na tabela 3 são apresentadas as porcentagens de pessoas de cada raça ou cor da pele de acordo com grupos de anos de escolaridade. Entre as pessoas brancas, $40,4 \%$ têm oito ou mais anos de escolaridade, mas entre as pessoas pretas essa porcentagem baixa a $22,9 \%$, praticamente igual à das pessoas pardas, que é $22,5 \%$ e bastante similar à dos indígenas, que é um pouco maior e alcança $26,8 \%$. Já para pessoas de raça amarela a porcentagem é de quase $61 \%$. Para 15 anos e mais, o que corresponde (quase certamente) a estudos universitários, a porcentagem nacional é de $4,4 \%$, mas entre pessoas negras e pardas é de apenas $1,3 \%$ com $6,7 \%$ para pessoas

\section{Figura 1}

Curvas de concentração de pessoas, de acordo com a raça ou cor da pele, para renda per capita, Brasil, 1998.

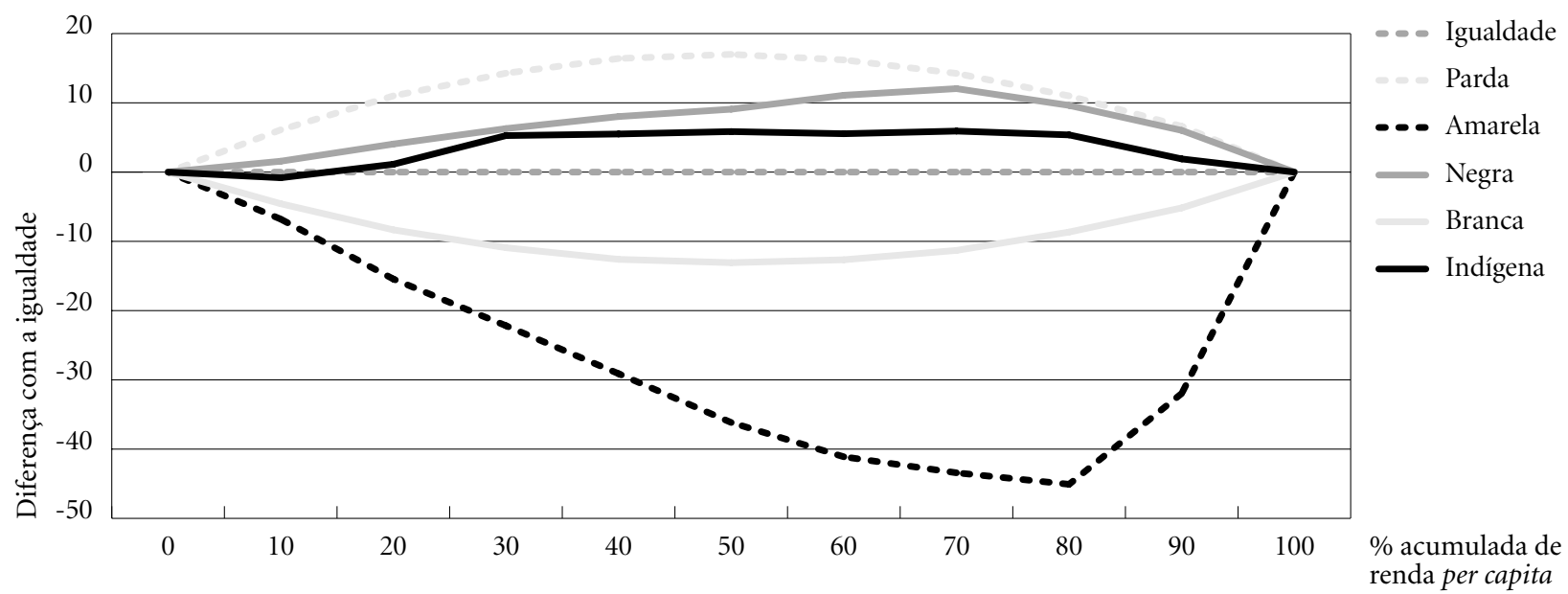

Tabela 3

Porcentagens de pessoas de cada raça ou cor da pele, de acordo com grupos de anos de escolaridade, Brasil, 1998.

\begin{tabular}{lrrrrrr}
\hline \multirow{2}{*}{ Anos de estudo } & \multicolumn{5}{c}{ Cor da pele ou raça } & \multicolumn{2}{c}{ Total } \\
& Indígena & Branca & Preta & Amarela & Parda & \\
\hline Menos de um ano & 16,35 & 9,19 & 21,67 & 5,84 & 19,98 & 14,08 \\
1 a 3 anos & 20,45 & 15,52 & 22,32 & 8,40 & 24,16 & 19,22 \\
4 a 7 anos & 36,39 & 34,90 & 33,11 & 24,79 & 33,40 & 34,16 \\
8 a 10 anos & 10,54 & 16,32 & 12,81 & 16,30 & 12,07 & 14,46 \\
11 a 14 anos & 11,73 & 17,34 & 8,75 & 26,54 & 9,07 & 13,68 \\
15 anos e mais & 4,53 & 6,74 & 1,33 & 18,13 & 1,32 & 4,39 \\
Total & 100,00 & 100,00 & 100,00 & 100,00 & 100,00 & 100,00 \\
\hline
\end{tabular}

Fonte: PNAD/1998 
brancas e $18,1 \%$ para pessoas de raça amarela. Para pessoas negras e pardas, aproximadamente uma de cada cinco estão na categoria de menos de um ano, que inclui nunca ter estado na escola.

A composição racial e de cor da pele das grandes regiões é bastante diferente como se pode apreciar da tabela 4 . Cerca de $54 \%$ da população brasileira se declara de cor branca, mas as regiões Norte e Nordeste têm apenas cerca de $30 \%$ de população branca, com predominância de cor parda com $68,5 \%$ e $64,3 \%$, respectivamente. Na região Sudeste, a população de cor branca chega aos $64 \%$, e no Sul alcança quase $83 \%$. A região Centro-Oeste é a única em que essas porcentagens são bastante parecidas.
A distribuição da população de acordo com anos de escolaridade é também bastante diferente de acordo com as grandes regiões do país, como se vê na tabela 5. No Nordeste mais de $48 \%$ da população de 15 anos e mais têm três anos ou menos de escolaridade, mas essa porcentagem é de cerca de $23 \%$ nas regiões Sul e Sudeste. No Sudeste, quase $44 \%$, e no Sul, cerca de $39 \%$, têm 8 anos ou mais de escolaridade, mas no Nordeste a porcentagem é de apenas cerca de $26 \%$. Nesta última região existem mais pessoas de 15 anos de idade e mais que têm menos de um ano de escolaridade do que as que têm oito ou mais.

Apesar desse quadro escandaloso da baixa escolaridade no Brasil, o panorama tem melho-

Tabela 4

Distribuição de raça e cor da pele da população das grandes regiões, Brasil, 1998.

\begin{tabular}{lcccccc}
\hline Grandes regiões & \multicolumn{2}{c}{ Cor da pele ou raça } & \multicolumn{2}{c}{ Total } \\
& Indígena & Branca & Preta & Amarela & Parda & 100,00 \\
Norte & 0,39 & 28,67 & 2,23 & 0,24 & 68,49 & 100,00 \\
Nordeste & 0,14 & 29,71 & 5,74 & 0,09 & 64,31 & 100,00 \\
Sudeste & 0,21 & 64,04 & 7,31 & 0,93 & 27,52 & 100,00 \\
Sul & 0,22 & 82,91 & 3,03 & 0,34 & 13,49 & 100,00 \\
Centro-Oeste & 0,62 & 46,87 & 3,68 & 0,50 & 48,32 & 100,00 \\
Total & 0,23 & 54,01 & 5,69 & 0,53 & 39,54 & \\
\hline
\end{tabular}

Fonte: PNAD/1998

\section{Tabela 5}

Distribuição da população de 15 anos e mais nas grandes regiões

e por grupos de idade, de acordo com grupos de anos de escolaridade, Brasil, 1998.

\begin{tabular}{|c|c|c|c|c|c|c|c|}
\hline & \multicolumn{6}{|c|}{ Anos de Estudo } & \multirow[t]{2}{*}{ Total } \\
\hline & Menos de 1 ano & 1 a 3 & 4 a 7 & 8 a 10 & 11 a 14 & 15 e mais & \\
\hline \multicolumn{8}{|l|}{ Grandes regiões } \\
\hline Norte & 15,77 & 16,03 & 31,27 & 17,44 & 16,50 & 3,00 & 100,00 \\
\hline Nordeste & 27,57 & 20,29 & 26,36 & 11,09 & 11,99 & 2,70 & 100,00 \\
\hline Sudeste & 9,89 & 13,29 & 32,93 & 19,30 & 17,98 & 6,61 & 100,00 \\
\hline Sul & 9,25 & 14,09 & 37,86 & 17,76 & 15,74 & 5,29 & 100,00 \\
\hline Centro-Oeste & 12,69 & 14,69 & 34,49 & 17,03 & 15,97 & 5,14 & 100,00 \\
\hline \multicolumn{8}{|l|}{ Grupos de idade } \\
\hline 15 a 44 anos & 8,57 & 13,00 & 34,05 & 20,74 & 18,80 & 4,84 & 100,00 \\
\hline 45 a 64 anos & 23,83 & 21,27 & 29,16 & 8,33 & 10,66 & 6,76 & 100,00 \\
\hline 65 a 84 anos & 43,47 & 21,62 & 22,64 & 4,69 & 5,03 & 2,56 & 100,00 \\
\hline 85 anos e mais & 58,77 & 15,75 & 15,91 & 3,47 & 4,04 & 2,06 & 100,00 \\
\hline Total & 15,08 & 15,55 & 31,94 & 16,58 & 15,79 & 5,07 & 100,00 \\
\hline
\end{tabular}

Fonte: PNAD/1998 
rado ao longo do tempo, o que se pode apreciar das porcentagens da segunda parte da tabela 5 na qual se mostra a distribuição das pessoas de 15 anos de idade e mais segundo grupos de anos de escolaridade por grupos de idade. Nos grupos de mais idade a situação é ainda pior. Por exemplo, enquanto no grupo de 65 a 84 anos de idade mais de $65 \%$ das pessoas têm apenas três ou menos anos de escolaridade, essa porcentagem é de somente cerca de $22 \%$ para as pessoas de 15 a 44 anos de idade. Esse aumento no número de anos de escolaridade foi, no entanto, bastante desigual de acordo com as grandes regiões do país. Na tabela 6, mostram-se as distribuições de escolaridade para a população de 15 anos de idade e mais segundo as grandes regiões para dois grupos de idade, de 15 a 44 e de 65 a 84 anos de idade. Não se levou em conta a migração entre as regiões, de forma que as porcentagens são de pessoas residentes em cada grande região na ocasião da pesquisa. Poderia ocorrer que as pessoas que migraram tivessem uma distribuição de anos de escolaridade diferente daquelas que não o fizeram. Os dados da PNAD permitem estudar esse aspecto em detalhe e profundidade, mas escapa ao escopo deste relatório em que a concentração é sobre estado de saúde e gasto em saúde.

Observa-se, por exemplo, que no Nordeste a porcentagem de pessoas com menos de um ano de instrução, que no grupo de 65 a 84 anos de idade é de quase $64 \%$, baixa a cerca de $18 \%$ no grupo de 15 a 44 anos, enquanto no Sudeste e no Sul cai de cerca de $34 \%$ e $32 \%$, respectivamente, a menos de $5 \%$. A porcentagem de pessoas com 8 anos de escolaridade ou mais, que é de apenas cerca de 7\% no Nordeste e próxima a $16 \%$ no Sudeste e a $12 \%$ no Sul para as pessoas de 65 a 84 anos de idade, alcança quase $31 \%$ no Nordeste, pouco mais de $52 \%$ no Sudeste e é ligeiramente superior a $47 \%$ no Sul para pessoas de 15 a 44 anos de idade.

A relação entre escolaridade e colocação da pessoa na distribuição de renda é muito forte no Brasil, o que era de esperar frente à grande desigualdade existente nas duas variáveis. A tabela 7 apresenta a distribuição de pessoas segundo quintis de renda per capita e grupos de anos de escolaridade. Estão excluídas da tabela as pessoas de menos de 15 anos de idade que não poderiam ter completado os respectivos números de anos de escolaridade e por esta razão as porcentagens em cada quintil não são de $20 \%$. Além disso, a renda nesses casos depende geralmente dos adultos do domicílio e de suas condições de trabalho. Alguns exemplos dessa fortíssima associação:

- Das pessoas do quintil de renda per capita mais baixo, quase $92 \%$ têm menos de oito anos

Tabela 6

Distribuição da população de dois grupos de idade nas grandes regiões, de acordo com grupos de anos de escolaridade, Brasil, 1998.

\begin{tabular}{|c|c|c|c|c|c|c|c|}
\hline \multirow[t]{2}{*}{ Grandes Regiões } & \multicolumn{6}{|c|}{ Anos de Estudo } & \multirow[t]{2}{*}{ Total } \\
\hline & Menos de 1 ano & 1 a 3 & 4 a 7 & 8 a 10 & 11 a 14 & 15 e mais & \\
\hline \multirow{2}{*}{\multicolumn{8}{|c|}{$\begin{array}{l}\text { Grupo de } 15 \text { a } \\
44 \text { anos de idade }\end{array}$}} \\
\hline & & & & & & & \\
\hline Norte & 9,17 & 13,97 & 34,53 & 20,55 & 18,92 & 2,86 & 100,00 \\
\hline Nordeste & 17,97 & 20,18 & 31,05 & 14,06 & 14,24 & 2,51 & 100,00 \\
\hline Sudeste & 4,60 & 9,78 & 33,49 & 24,27 & 21,48 & 6,39 & 100,00 \\
\hline Sul & 4,13 & 9,61 & 38,93 & 22,73 & 19,34 & 5,26 & 100,00 \\
\hline Centro-Oeste & 6,35 & 11,95 & 38,21 & 20,38 & 18,42 & 4,70 & 100,00 \\
\hline Total & 8,57 & 13,00 & 34,05 & 20,74 & 18,80 & 4,84 & 100,00 \\
\hline \multirow{2}{*}{\multicolumn{8}{|c|}{$\begin{array}{l}\text { Grupo de } 65 \text { a } \\
84 \text { anos de idade }\end{array}$}} \\
\hline & & & & & & & \\
\hline Norte & 51,93 & 22,44 & 15,53 & 4,45 & 4,41 & 1,24 & 100,00 \\
\hline Nordeste & 63,55 & 18,15 & 11,69 & 2,52 & 2,71 & 1,37 & 100,00 \\
\hline Sudeste & 34,19 & 22,16 & 27,70 & 5,89 & 6,75 & 3,31 & 100,00 \\
\hline Sul & 32,05 & 26,01 & 30,11 & 4,83 & 4,32 & 2,68 & 100,00 \\
\hline Centro-Oeste & 50,15 & 21,42 & 16,76 & 5,03 & 4,11 & 2,52 & 100,00 \\
\hline Total & 43,47 & 21,62 & 22,64 & 4,69 & 5,03 & 2,56 & 100,00 \\
\hline
\end{tabular}

Fonte: PNAD/1998 
Tabela 7

Distribuição de população, segundo quintis de renda per capita e grupos de anos de escolaridade, Brasil, 1998. (Em cada célula os números são, respectivamente, porcentagem na linha e porcentagem na coluna).

\begin{tabular}{|c|c|c|c|c|c|c|c|}
\hline \multirow{2}{*}{$\begin{array}{l}\text { Quintis de } \\
\text { renda per capita }\end{array}$} & \multirow[b]{2}{*}{ Menos de 1 ano } & \multirow[b]{2}{*}{1 a 3} & \multicolumn{2}{|c|}{ Anos de Estudo } & \multirow[b]{2}{*}{11 a 14} & \multirow[b]{2}{*}{15 e mais } & \multirow[t]{2}{*}{ Total } \\
\hline & & & 4 a 7 & 8 a 10 & & & \\
\hline \multirow[t]{2}{*}{1} & 27,72 & 33,25 & 30,92 & 5,85 & 2,12 & 0,14 & 100,00 \\
\hline & 33,5 & 29,28 & 15,36 & 6,90 & 2,65 & 0,58 & 17,03 \\
\hline \multirow[t]{2}{*}{2} & 20,03 & 25,84 & 38,88 & 10,41 & 4,67 & 0,17 & 100,00 \\
\hline & 27,38 & 25,75 & 21,85 & 13,88 & 6,63 & 0,76 & 19,27 \\
\hline \multirow[t]{2}{*}{3} & 15,71 & 19,71 & 40,21 & 15,22 & 8,70 & 0,45 & 100,00 \\
\hline & 22,89 & 20,93 & 24,09 & 21,63 & 13,14 & 2,18 & 20,54 \\
\hline \multirow[t]{2}{*}{4} & 7,82 & 14,58 & 37,70 & 20,36 & 17,75 & 1,79 & 100,00 \\
\hline & 11,68 & 15,88 & 23,15 & 29,66 & 27,49 & 8,88 & 21,05 \\
\hline \multirow[t]{2}{*}{5} & 2,90 & 7,14 & 24,09 & 18,26 & 30,79 & 16,82 & 100,00 \\
\hline & 4,55 & 8,16 & 15,54 & 27,94 & 50,09 & 87,59 & 22,11 \\
\hline \multirow[t]{2}{*}{ Total } & 14,09 & 19,34 & 34,28 & 14,45 & 13,59 & 4,25 & 100,00 \\
\hline & 100,00 & 100,00 & 100,00 & 100,00 & 100,00 & 100,00 & 100,00 \\
\hline
\end{tabular}

Fonte: PNAD/1998

de escolaridade. Já no quintil de renda mais alto, apenas $34,1 \%$ têm 7 anos ou menos de escolaridade;

- Menos de $11 \%$ das pessoas com menos de um ano de escolaridade estão nos dois quintis de renda per capita mais alta;

- Para as pessoas com 15 anos de escolaridade e mais, quase $90 \%$ estão no quintil de renda per capita mais alto;

- Das pessoas do quintil de renda per capita mais baixo, quase $92 \%$ têm menos de oito anos de escolaridade. Já no quintil de renda mais alto, apenas $34,1 \%$ têm 7 anos ou menos de escolaridade.

Para as características de saúde, foi captado se as informações da pessoa pesquisada foram prestadas pela própria, por outro morador ou por não-morador da unidade domiciliar. Para a auto-avaliação do estado de saúde da pessoa, avaliado segundo seu próprio ponto de vista, ou, no caso de criança pequena, do ponto de vista do seu responsável, foi classificado em uma escala de cinco graus: muito bom, bom, regular, ruim ou muito ruim.

Segundo os dados da pesquisa, expandidos, para pessoas de 15 anos de idade e mais, em $50,2 \%$ dos casos a auto-avaliação foi da própria pessoa e em $47,4 \%$ por outro morador do domicílio. Em 2,4\% dos casos, a avaliação foi feita por outra pessoa, não moradora no domicílio.
Existem diferenças nos resultados, dependendo de se era realmente uma auto-avaliação ou a opinião sobre outro indivíduo.

A tabela 8 mostra as porcentagens de classificações nas cinco categorias de acordo com quem fez a avaliação. Em termos de classificação percentual nas cinco categorias, a avaliação de não moradores é muito próxima à auto-avaliação pela própria pessoa; mas quando a avaliação é feita por outro morador do próprio domicílio, este tende a classificar em categorias melhores que a própria pessoa. Para os casos de autoclassificação, 68,5\% das pessoas colocam seu estado de saúde como muito bom e bom, mas esta porcentagem sobe a 79,1\% quando a classificação foi feita por outro morador. Pessoas que se autoclassificam escolhem ruim ou muito ruim em 7,0\% das vezes, mas quando a classificação é feita por outro morador essa porcentagem baixa a cerca da metade, para 3,6\%.

Infelizmente o fato de ser uma autoclassificação ou uma classificação por outro morador está relacionado com o nível de renda. Na primeira parte da tabela 9, apresenta-se a porcentagem de classificações pela própria pessoa, outro morador ou não morador, para pessoas de 15 anos e mais, segundo decis de renda per capita. No decil de renda per capita mais baixo, a porcentagem de autoclassificações é de 55,6\%, caindo a $48,8 \%$ no decil de renda mais alto. $\mathrm{Pa}$ - 
Tabela 8

Distribuição da avaliação de saúde de pessoas de

15 anos e mais, de acordo com quem fez a avaliação, Brasil, 1998.

\begin{tabular}{lcccr}
\hline \multirow{2}{*}{$\begin{array}{l}\text { Classificação do } \\
\text { estado de saúde }\end{array}$} & \multicolumn{2}{c}{ Quem informou sobre o estado de saúde } & \multirow{2}{*}{ Total } \\
\hline Muito bom & A própria pessoa & Outro morador & Não morador & \\
Bom & 21,85 & 25,95 & 19,34 & 23,74 \\
Regular & 46,61 & 53,11 & 49,89 & 49,77 \\
Ruim & 25,54 & 17,29 & 23,87 & 21,59 \\
Muito ruim & 5,06 & 2,91 & 5,68 & 4,06 \\
Não informado & 0,92 & 0,70 & 1,21 & 0,82 \\
Total & 0,01 & 0,03 & 0,00 & 0,02 \\
\end{tabular}

Fonte: PNAD/1998

Tabela 9

Porcentagens de casos em que a classificação do estado de saúde foi feita pela própria pessoa, por outro morador do domicílio ou por uma pessoa não moradora no mesmo, para pessoas de 15 anos de idade e mais, segundo decis de renda per capita e para as grandes regiões, Brasil, 1998.

\begin{tabular}{lcccc}
\hline \multicolumn{4}{c}{ Quem informou sobre o estado de saúde } & Total \\
& A própria pessoa & Outro morador & Não morador & \\
\hline $\begin{array}{l}\text { Por decis de } \\
\text { renda per capita }\end{array}$ & & & \\
1 & 56,56 & 41,56 & 1,88 & 100,00 \\
2 & 53,90 & 44,59 & 1,51 & 100,00 \\
3 & 52,72 & 45,38 & 1,89 & 100,00 \\
4 & 50,30 & 47,76 & 1,94 & 100,00 \\
5 & 49,51 & 48,51 & 1,99 & 100,00 \\
6 & 53,20 & 43,30 & 3,50 & 100,00 \\
7 & 48,06 & 49,34 & 2,60 & 100,00 \\
8 & 47,43 & 49,79 & 2,78 & 100,00 \\
9 & 47,76 & 49,83 & 2,42 & 100,00 \\
10 & 48,82 & 49,06 & 2,13 & 100,00 \\
Para as grandes & & & & \\
regiões do país & & & & 100,00 \\
Norte & 50,68 & 47,50 & 1,82 & 100,00 \\
Nordeste & 53,62 & 44,38 & 2,00 & 100,00 \\
Sudeste & 47,41 & 49,95 & 2,64 & 100,00 \\
Sul & 51,67 & 45,92 & 2,41 & 100,00 \\
Centro-Oeste & 52,04 & 45,89 & 2,07 & 100.00 \\
Total & 50,47 & 47,21 & 2,32 & \\
\hline
\end{tabular}

Fonte: PNAD/1998 
ra de grandes regiões, as variações são menores mas não desprezíveis, como se pode observar na segunda parte da tabela 9 . O fato de se ter maiores porcentagens de autoclassificação em níveis de rendas mais baixas e em regiões menos desenvolvidas poderia relacionar-se com fatores de mercado de trabalho, já que a maior porcentagem de autoclassificações se associa ao fato de as pessoas estarem no domicílio na ocasião da entrevista do inquérito.

A relação do estado de saúde com os níveis de renda é no entanto muito mais forte do que seria possível explicar apenas por variações entre ter sido auto-avaliado ou classificado por outra pessoa, moradora ou não no domicílio. Isso se pode apreciar na tabela 10 . A porcentagem de classificações na categoria muito bom sobe de $28,5 \%$ no decil de renda mais baixo a $39,1 \%$ no decil de renda mais alto, enquanto a porcentagem de classificações ruim e muito ruim cai de $3,7 \%$ a $1,6 \%$. Cerca de $50 \%$, com pouca variação por nível de renda, são classificações na categoria de bom.

A classificação do estado de saúde também varia de acordo com as grandes regiões do país e com a raça ou cor de pele da pessoa (segunda e terceira partes da tabela 10), mas as maiores variações estão associadas com o nível de escolaridade (Tabela 11).

Para nível de educação formal as diferenças chegam a ser dramáticas. No grupo de pessoas sem nenhuma instrução ou menos de um ano de escola, a porcentagem de classificações como ruim é de $11,3 \%$ e como muito ruim, de 2,2\%. No grupo de 15 anos ou mais, a soma das porcentagens nessas duas classificações é de somente $1,1 \%$. No grupo sem instrução ou menos de um ano de escola, apenas $51,8 \%$ se classificam

Tabela 10

Porcentagem de pessoas classificadas de acordo com seu estado de saúde, por decis de renda per capita, nas grandes regiões e de acordo com a raça ou cor da pele, Brasil, 1998.

\begin{tabular}{|c|c|c|c|c|c|c|}
\hline & \multicolumn{5}{|c|}{ Classificação do estado de saúde } & \multirow[t]{2}{*}{ Total } \\
\hline & Muito bom & Bom & Regular & Ruim & Muito ruim & \\
\hline \multicolumn{7}{|c|}{$\begin{array}{l}\text { Segundo decis } \\
\text { de renda per capita }\end{array}$} \\
\hline 1 & 28,53 & 51,37 & 16,4 & 3,15 & 0,50 & 100,00 \\
\hline 2 & 26,43 & 51,58 & 17,83 & 3,46 & 0,66 & 100,00 \\
\hline 3 & 23,79 & 52,25 & 19,16 & 4,02 & 0,77 & 100,00 \\
\hline 4 & 25,3 & 51,07 & 19,09 & 3,85 & 0,67 & 100,00 \\
\hline 5 & 26,01 & 52,30 & 18,04 & 3,05 & 0,59 & 100,00 \\
\hline 6 & 24,00 & 48,57 & 21,49 & 4,92 & 1,00 & 100,00 \\
\hline 7 & 27,81 & 51,34 & 17,63 & 2,55 & 0,63 & 100,00 \\
\hline 8 & 28,49 & 51,64 & 17,04 & 2,19 & 0,60 & 100,00 \\
\hline 9 & 32,95 & 50,38 & 14,40 & 1,81 & 0,44 & 100,00 \\
\hline 10 & 39,05 & 47,83 & 11,50 & 1,28 & 0,33 & 100,00 \\
\hline \multicolumn{7}{|l|}{$\begin{array}{l}\text { Por grandes } \\
\text { regiões do país }\end{array}$} \\
\hline Norte & 17,69 & 56,64 & 21,29 & 3,64 & 0,74 & 100,00 \\
\hline Nordeste & 28,15 & 48,6 & 18,70 & 3,88 & 0,67 & 100,00 \\
\hline Sudeste & 30,98 & 50,5 & 15,54 & 2,45 & 0,53 & 100,00 \\
\hline Sul & 26,01 & 52,81 & 17,47 & 3,02 & 0,69 & 100,00 \\
\hline Centro-Oeste & 23,99 & 54,18 & 18,45 & 2,63 & 0,76 & 100,00 \\
\hline \multicolumn{7}{|l|}{$\begin{array}{l}\text { Segundo raça } \\
\text { ou cor da pele }\end{array}$} \\
\hline Indígena & 23,81 & 57,53 & 15,48 & 2,63 & 0,55 & 100,00 \\
\hline Branca & 30,49 & 50,00 & 16,21 & 2,70 & 0,60 & 100,00 \\
\hline Preta & 27,31 & 49,57 & 18,59 & 3,78 & 0,75 & 100,00 \\
\hline Amarela & 29,57 & 53,96 & 13,88 & 2,09 & 0,50 & 100,00 \\
\hline Parda & 25,32 & 52,15 & 18,52 & 3,37 & 0,65 & 100,00 \\
\hline Total & 28,25 & 50,86 & 17,24 & 3,02 & 0,62 & 100,00 \\
\hline
\end{tabular}

Fonte: PNAD/1998 
em muito bom e bom, enquanto para pessoas com 15 anos e mais de estudo essa porcentagem é de 90,1\%.

É importante examinar como se comporta a variável em relação à idade. É também importante considerar que quem informa sobre o estado de saúde é diferente de acordo com a idade. Na tabela 12 mostram-se as porcentagens de casos em que o informante foi a própria pessoa, um outro morador do domicílio ou um não morador, de acordo com os grupos de idade. Existem algumas inconsistências, como pequenas porcentagens de crianças menores de 5 anos (e até de um ano) que informaram sobre sua própria saúde. Para os grupos de 15 anos e mais, a porcentagem de informação pela própria pessoa sobe de $45,6 \%$ no grupo de 15 a 44 anos até $65,9 \%$ no grupo de 65 a 84, caindo para $39,5 \%$ no grupo de 85 anos de idade e mais. Nesse último grupo a porcentagem de infor- mação por um não morador passa também a ser relevante, é de $8,8 \%$.

Como seria de esperar, as porcentagens de classificação no estado de saúde muito bom caem com a idade. A porcentagem de muito bom passa de 40,4\% nos menores de um ano e $38,5 \%$ no grupo de 1 a 4 anos a $13,1 \%$ no grupo de 45 a 64 anos, 5,7\% no grupo de 65 a 84 e é de somente $4,6 \%$ para pessoas de 85 anos de idade e mais. O inverso ocorre para as classificações ruim e muito ruim. São, juntas, menos de $1 \%$ até o grupo de 5 a 14 anos de idade, pouco mais de $2 \%$ no grupo de 15 a 44 anos e sobem a $8,7 \%$ para pessoas de 45 a 64 anos, $17,8 \%$ para as de 65 a 84 , alcançando o valor de $27,4 \%$ para aquelas de 85 anos de idade e mais. Em todos os grupos de idade se observa o aumento da porcentagem de classificações no estado de saúde muito bom, de acordo com o aumento do nível de renda. Para os estados de saúde ruim e muito ruim

Tabela 11

Porcentagem de pessoas classificadas de acordo com seu estado de saúde, para idades de 15 anos e mais, de acordo com anos de estudo, Brasil, 1998.

\begin{tabular}{lccrrrr}
\hline \multirow{2}{*}{ Anos de escolaridade } & \multicolumn{2}{c}{ Classificação do estado de saúde } & \multicolumn{2}{c}{ Total } \\
& Muito bom & Bom & Regular & Ruim & Muito ruim & \\
\hline Menos de um ano & 13,17 & 38,67 & 34,63 & 11,32 & 2,21 & 100,00 \\
1 a 3 anos & 23,12 & 49,18 & 22,55 & 4,34 & 0,80 & 100,00 \\
4 a 7 anos & 25,93 & 52,65 & 18,53 & 2,35 & 0,55 & 100,00 \\
8 a 10 anos & 30,03 & 54,38 & 13,96 & 1,30 & 0,32 & 100,00 \\
11 a 14 anos & 32,62 & 54,1 & 12,01 & 1,04 & 0,23 & 100,00 \\
15 anos e mais & 39,82 & 50,25 & 8,83 & 0,87 & 0,23 & 100,00 \\
Total & 25,71 & 50,37 & 19,58 & 3,59 & 0,74 & 100,00 \\
\hline
\end{tabular}

Fonte: PNAD/1998

\section{Tabela 12}

Porcentagens de casos em que a classificação do estado de saúde foi feita pela própria pessoa, por outro morador do domicílio ou por uma pessoa não moradora no mesmo, por grupos de idade, Brasil, 1998.

\begin{tabular}{lcccc}
\hline Grupos de idade & \multicolumn{2}{c}{ Quem informou sobre o estado de saúde } & Total \\
& A própria pessoa & Outro morador & Não morador & \\
\hline Menos de um ano & 0,38 & 97,62 & 2,00 & 100,00 \\
de 1 a 4 anos & 0,58 & 97,18 & 2,24 & 100,00 \\
de 5 a 14 anos & 7,69 & 90,46 & 1,86 & 100,00 \\
de 15 a 44 anos & 45,62 & 52,23 & 2,15 & 100,00 \\
de 45 a 64 anos & 59,27 & 38,50 & 2,23 & 100,00 \\
de 65 a 84 anos & 65,87 & 30,21 & 3,92 & 100,00 \\
de 85 anos e mais & 39,52 & 51,67 & 8,81 & 100,00 \\
Total & 36,79 & 60,97 & 2,23 & 100,00 \\
\hline
\end{tabular}

Fonte: PNAD/1998 
ocorre o inverso, com gradientes maiores com o aumento da idade. Em outras palavras, as pessoas de baixos níveis de renda que sobrevivem até idades mais avançadas vivem em um estado de saúde muito pior do que as pessoas de níveis mais altos de renda. Os dois gráficos da figura 2 apresentam um resumo dessas observações para a classificação de estado de saúde muito bom no primeiro caso e para as classificações ruim e muito ruim combinadas, no segundo.

Para a variável "estado de saúde muito bom”, com valores 1 para sim e 0 não, os resultados finais da regressão logística são apresentados na tabela 13. O indicador de "informado por outra pessoa do mesmo domicílio", que isoladamente tinha uma razão de produtos cruzados 1,577, com intervalo de confiança de $95 \%$ de 1,488 a 1,670 , sai do ajuste ao introduzir-se $\mathrm{o}$ indicador de sexo feminino. Existe uma forte relação entre o sexo da pessoa sobre a qual está sendo informada e quem deu a informação. Para os homens, somente $26,5 \%$ informaram sobre seu próprio estado de saúde, sendo esta informação dada por outro morador em $71,18 \%$ das vezes. Já para as mulheres, 46,58\% delas classificaram seu próprio estado de saúde e em $51,16 \%$ das vezes o informante foi outro morador. O informante foi um não morador para $2,21 \%$ dos homens e $2,26 \%$ das mulheres. Nenhum dos indicadores de raça ou cor da pele tem coeficiente estatisticamente significante depois de introduzidas as variáveis de anos de escolaridade e de decis de renda. Existe uma diferença significante para as áreas rurais nas quais a probabilidade de uma pessoa, após controlar para todas as demais variáveis, tem uma probabilidade maior de ser classificada como tendo estado de saúde muito bom.

Como se vê na tabela 14, a situação é em quase tudo similar para a variável "estado de saúde ruim ou muito ruim”, mas o indicador de informado por outro morador do próprio domicílio permanece no modelo. Após controlar por todas as demais variáveis, a probabilidade de uma pessoa ser classificada como tendo saúde ruim ou muito ruim é significante menor nas áreas rurais.

\section{Conclusões e comentários}

É importante ter sempre presente que o que se está estudando são determinações do estado de saúde autopercebido e não de uma medida "objetiva” do mesmo. Essa distinção é muito impor-
Figura 2

Porcentagem de pessoas que tiveram seu estado de saúde classificado como (a) muito bom e (b) ruim e muito ruim, por grupos de idade, de acordo com os decis de renda per capita, Brasil, 1998.

Figura 2a



Figura 2b

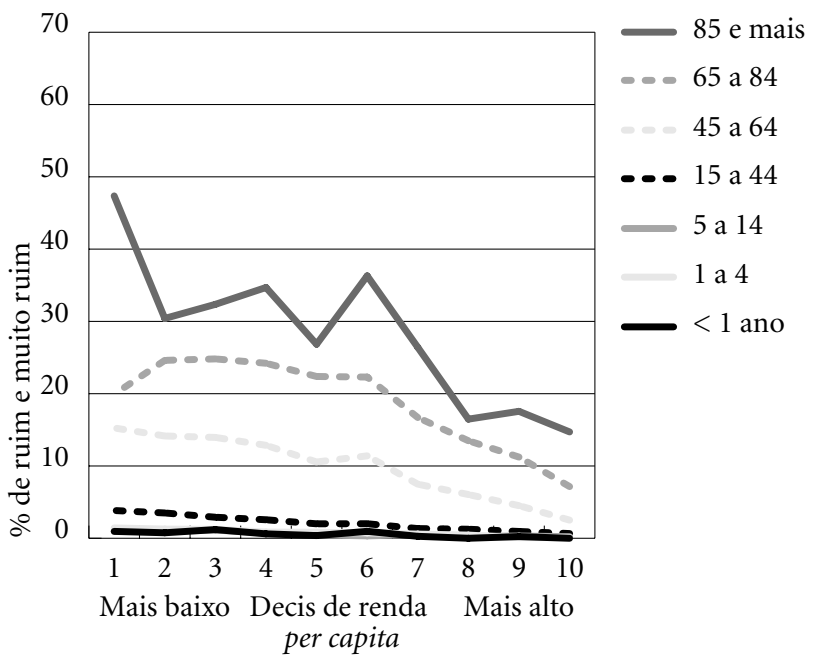

Fonte: PNAD/1998 
Tabela 13

Regressão logística para a probabilidade de uma pessoa ser classificada como tendo estado de saúde muito bom, de acordo com grupos de idade, anos de escolaridade, decis de renda per capita, sexo e área de residência, Brasil, 1998.

\begin{tabular}{lcccccc}
\hline Saúde muito boa & $\begin{array}{c}\text { Razão de } \\
\text { chances }\end{array}$ & Erro padrão & $\mathbf{t}$ & $\mathbf{P}>|\mathbf{t}|$ & \multicolumn{2}{c}{$\begin{array}{c}\text { [Intervalo de } \\
\text { conf. 95\%] }\end{array}$} \\
\hline Idade de 1 a 4 anos & 0,918 & 0,0339 & $-2,321$ & 0.021 & 0,854 & 0,987 \\
Idade de 5 a 14 anos & 0,812 & 0,0307 & $-5,497$ & 0.000 & 0,754 & 0,875 \\
Idade de 15 a 44 anos & 0,380 & 0,0182 & -20.154 & 0.000 & 0,346 & 0,418 \\
Idade de 45 a 64 anos & 0,143 & 0,0075 & -37.160 & 0.000 & 0,129 & 0,158 \\
Idade de 65 a 84 anos & 0,063 & 0,0042 & -41.422 & 0.000 & 0,055 & 0,072 \\
Idade de 85 anos e mais & 0,053 & 0,0082 & -19.137 & 0.000 & 0,039 & 0,072 \\
Anos de escolaridade & 1,055 & 0,0032 & 17,782 & 0.000 & 1,049 & 1,061 \\
Decis de renda per capita & 1,082 & 0,0081 & 10,603 & 0.000 & 1,066 & 1,098 \\
Sexo feminino & 0,852 & 0,0082 & -16.608 & 0.000 & 0,836 & 0,868 \\
Área rural & 1,278 & 0,0807 & 3,872 & 0.000 & 1,128 & 1,446 \\
\hline
\end{tabular}

Fonte: PNAD/1

$\mathrm{F}(10,382)=379.02$

Prob $>\mathrm{F}=0.0000$

Tabela 14

Regressão logística para a probabilidade de uma pessoa ser classificada como tendo estado de saúde ruim ou muito ruim, de acordo com quem deu a informação, grupos de idade, anos de escolaridade, decis de renda per capita, sexo e área de residência, Brasil, 1998.

\begin{tabular}{lccccrr}
\hline $\begin{array}{l}\text { Saúde ruim } \\
\text { ou muito ruim }\end{array}$ & Razão de & $\begin{array}{c}\text { Erro padrão } \\
\text { chances }\end{array}$ & $\mathbf{t}$ & $\mathbf{P}>|\mathbf{t}|$ & \multicolumn{1}{c}{$\begin{array}{c}\text { [Intervalo de } \\
\text { conf. 95\%] }\end{array}$} \\
\hline Outra pess. mesmo dom. & 0,868 & 0,0207 & $-5,930$ & 0,000 & 0,828 & 0,910 \\
Idade de 1 a 4 anos & 1,418 & 0,2437 & 2,033 & 0,043 & 1,012 & 1,988 \\
Idade de 5 a 14 anos & 1,374 & 0,2283 & 1,911 & 0,057 & 0,991 & 1,905 \\
Idade de 15 a 44 anos & 6,990 & 1,1812 & 11,506 & 0,000 & 5,014 & 9,744 \\
Idade de 45 a 64 anos & 25,761 & 4,2650 & 19,624 & 0,000 & 18,604 & 35,672 \\
Idade de 65 a 84 anos & 49,718 & 8,1561 & 23,813 & 0,000 & 36,012 & 68,642 \\
Idade de 85 anos e mais & 81,183 & 13,3788 & 26,679 & 0,000 & 58,716 & 112,248 \\
Anos de escolaridade & 0,866 & 0,0065 & $-19,262$ & 0,000 & 0,854 & 0,879 \\
Decis de renda per cap. & 0,899 & 0,0069 & $-13,899$ & 0,000 & 0,886 & 0,913 \\
Sexo feminino & 1,228 & 0,0306 & 8,219 & 0,000 & 1,169 & 1,290 \\
Área rural & 0,755 & 0,0321 & $-6,609$ & 0,000 & 0,694 & 0,821 \\
\hline
\end{tabular}

$\mathrm{F}(11,382)=1118,31$

Prob > F 0,0000

Fonte: PNAD 1998.

tante ao usar os resultados no desenho de políticas sociais que têm por objetivo diminuir as desigualdades observadas (Sen, 2002). Estão começando a surgir métodos para padronizar respostas a perguntas sobre autopercepção, sobre o estado de saúde (Tandon et al., 2002; van Doorslaer 2002) com resultados bastante promissores (Sadana et al., 2002). Em geral esses métodos dependem da disponibilidade de dados simultâneos sobre autopercepção e de um instrumento previamente calibrado, o que não existe no caso do Brasil, onde os estudos sobre a percepção sobre a saúde são praticamente inexistentes (Silveira, 2002).

Por outro lado o comportamento da autopercepção do estado geral de saúde no caso da população brasileira está muito próximo do que se conhece de outros estudos (Sadana et al., 2000 e 2002), e do que se poderia dizer é de se esperar num país com desigualdades socioeconômicas tão grandes. Essas desigualdades têm dimensões regionais importantes e se distribuem 
desigualmente de acordo com a escolaridade, a raça ou cor da pele das pessoas, e de acordo com a área urbana ou rural do país, com conseqüências importantes sobre a composição etária da população nesses diferentes grupos e áreas.

O determinante mais importante do estado de saúde autopercebido é a idade, mas ainda assim os níveis de educação e de rendimento apresentam contribuições adicionais grandes. A razão de chances de ser classificado no estado de saúde muito bom é 1,055 para cada ano adicional de escolaridade e de 1,082 para cada decil que se sobe na escala de rendimentos per capita. A probabilidade de ser classificada como com boa saúde é significantemente menor para as mulheres, mas a interpretação desse resultado está quase totalmente prejudicada pelo fato de a classificação ser diferente de acordo com quem faz a avaliação, sendo pior quando avaliada por outra pessoa do mesmo domicílio. Com relação aos homens, eles são avaliados por outra pessoa do próprio domicílio com maior freqüência do que as mulheres. Finalmente, na área rural é maior a probabilidade de que uma pessoa seja avaliada como com saúde muito boa, depois de controlar por todas as demais variáveis estudadas. Esse resultado é diferente do que se esperaria observar se a medida da auto-avaliação tivesse uma relação direta com o "estado real" de saúde das pessoas. O mais provável é que esse fato se deva a diferenças culturais relacionadas com menores níveis de informação e expectativas mais baixas em relação a seu próprio estado de saúde entre as pessoas das áreas rurais.

Para classificação em estado de saúde ruim ou muito ruim, os resultados são quase a imagem especular da situação anterior. A classificação de saúde auto-avaliada piora com a idade, de forma ainda mais acentuada que para saúde muito boa. Cada ano de escolaridade representa uma razão de chances de 0,866 de ser classificado em estado de saúde ruim ou muito ruim, uma relação mais forte que para saúde muito boa, o mesmo ocorrendo com a ascensão no nível de renda per capita, para o qual se obtém uma razão de chances de 0,899 para o avanço de um degrau nos decis de renda. Ao contrário da avaliação de saúde como muito boa, em que a educação apresenta uma influência marcadamente maior que o nível de renda, neste caso as duas variáveis têm efeitos similares. No caso de saúde ruim e muito ruim, as mulheres têm uma razão de chances de 1,288 comparadas com os homens e persiste a influência de quem faz a classificação. De novo, para populações em áreas rurais se observa uma relação contrária ao esperado, com uma razão de chances de 0,755 de forma que a probabilidade de uma pessoa ser classificada com saúde ruim ou muito ruim é marcadamente menor nessas áreas rurais que em áreas urbanas.

De maneira isolada, a cor da pele ou raça das pessoas está correlacionada com a avaliação do estado de saúde, mas, uma vez que se incluem os efeitos de escolaridade e renda, a cor da pele ou a raça deixam de ter efeitos estatisticamente significativos.

Não é nenhuma surpresa de que no Brasil os gradientes para a classificação em saúde muito boa ou em saúde ruim ou muito ruim de acordo com níveis de renda e especialmente de escolaridade são maiores do que em geral observado em outros países, dado que temos enormes desigualdades nesses determinantes na população do país. Por outro lado, a descoberta de que o comportamento na classificação do estado de saúde autopercebido é diferente nas áreas rurais em relação às áreas urbanas permite entender o comportamento aparentemente inexplicável de que nos decis inferiores de renda per capita as porcentagens de classificação de saúde muito boa sejam em geral um pouco maiores que nos níveis intermediários, para só a partir daí subirem de forma mais marcada para os decis superiores (Tabela 10) e para saúde ruim e muito ruim menores que nos níveis intermediários, só começando a cair a partir do terceiro decil de renda per capita. Este fato se relaciona com os níveis de renda mais baixos que se observam nas populações rurais.

$\mathrm{Da}$ análise feita e dos resultados encontrados, se extrai uma conclusão importantíssima para futuras pesquisas de saúde por meio de entrevista em inquéritos nacionais. É muito importante realizar um estudo independente que permita calibrar as perguntas de auto-avaliação do estado de saúde, através da aplicação simultânea de um instrumento de resultados comprovados como SF-36 ou Euroqol. Este estudo independente permitiria calibrar as respostas das áreas rurais em relação às áreas urbanas, bem como transformar essa variável ordinal numa outra contínua que permite mais amplitude na análise e interpretações mais seguras de relações de determinação, as quais, por sua vez, oferecem mais segurança no uso de resultados para a formulação de políticas (van Doorslaer e Jones, 2002).

Além disso, deve-se considerar seriamente a adoção de um procedimento diferente na ob- 
tenção dos dados para essa parte de um futuro módulo de saúde, devido ao problema observado de a distribuição da classificação de estado de saúde ser diferente de acordo com a fonte da informação, ou seja, se fornecida pela própria pessoa ou por outro morador do domicílio. Existem pelo menos duas alternativas possíveis. Uma delas consiste em exigir visitas sucessivas até que todos os maiores de 14 anos possam ser entrevistados sobre seu estado de saúde. A ou- tra é escolher ao acaso um dos moradores de 14 anos ou mais para dar informação sobre o seu próprio estado de saúde, e visitar o domicílio novamente se essa pessoa não esteve presente no momento de aplicar o restante do questionário. Ambas apresentam vantagens e desvantagens e implicações de custo, mas garantem que esta variável espúria não estará influenciando os resultados.

\section{Referências bibliográficas}

Almeida Filho N 1998. Desigualdades em saúde segundo condições de vida: análise da produção científica na América Latina e Caribe. Pan American Health Organization, Washington, D.C. Pesquisa em Saúde Pública, Documentos Técnicos No. 19. Disponível em <www. paho.org/Portuguese/HDP/HDR/série19 composite. pdf $>$.

Almeida Filho N, Kawachi I, Pellegrini A, Dachs N 2002. Research on health inequities in Latin America and the Caribbean: bibliometric and content analysis (19712000). Submetido para publicação.

Appels A, Bosma H, Grabauskas V, Gostautas A, Sturmans F 1996. Self-rated health and mortality in a Lithuanian and a Dutch population. Social Science and Medicine 42(5:681-689.

Banco Interamericano de Desenvolvimento 1999. Economic and social progress in Latin America, 1998/1999 report: Facing up to inequality in Latin America. Washington, DC: IADB.

Beckett M, Weinstein M, Goldman N, Yu-Hsuan L 2000. Do health interview surveys yield reliable data on chronic illness among older respondents? American Journal of Epidemiology 151(3):315-323.

Bollen KA, Glanville JL, Stecklov G 2001. Economic status proxies in studies of fertility in developing countries: does the measure matter? Measure Evaluation Working Paper 01-38. Disponível em <www. cpc.unc.edu/measure/publications/workingpapers/ wp0138.pdf $>$.

Casas JA, Dachs JNW, Bambas A 2001. Health disparities in Latin America and the Caribbean: the role of social and economic determinants. In equity and health: views from the Pan American Sanitary Bureau. Occasional Publication No. 8, pp.22-49. Pan American Health Organization, Washington, D.C. Disponível em <www.paho.org/English/DBI/Op08/ OP08_03.pdf $>$.
Dachs JNW 2001. Inequidades en salud: como estudiarlas. In Málaga H, Restrepo H (eds.). Promoción de la Salud: Construyendo comunidades saludables. Editorial Médica Panamericana, Bogotá, Colômbia.

Dachs JNW 2002. On the use of household surveys and other sources of information for monitoring health equity. Pan American Journal of Public Health (aceito para publicação).

Dachs JNW et al. 2002. Inequities in health in Latin America and the Caribbean: Descriptive and exploratory results for twelve contries. Pan American Journal of Public Health (aceito para publicação).

Deaton, A 1994. The analysis of household survey: a microeconometric approach to development policy. Johns Hopkins Un. Press, Baltimore. (World Bank Series).

Groot W 2000. Adaptation and scale of reference bias in self-assessment of quality of life. Journal of Health Economics 19:403-420.

Idler EL, Kasl SV, Lemke JH 1990. Self-evaluated health and mortality among the elderly in New Haven, Connecticut, and Iowa and Washington counties, Iowa, 1982-1986. American Journal of Epidemiology 131(1): 91-103.

Johnson TP et al. 1996. Cultural variations in the interpretation of health survey questions. In Warnecke R (ed.). Health survey research methods conference proceeding. DHSS Publication no. PHS 96-1.013, pp. 57-62.

Knäuper B, Belli RF, Herzog AR, Hill DH 1995. The quality of survey data as affected by question difficulty and respondents $=$ cognitive capacity. Journal of Official Statistics 13(2):181-99.

Kroeger A 1983. Health interview surveys in developing countries: a review of the methods and results. International Journal of Epidemiology 12(4):465-481.

Kroeger A 1985. Response errors and other problems of health interview surveys in developing countries. World Health Statistical Quarterly 38:15-33. 
Leung KK, Tang LY, Lue BH 1997. Self-rated health and mortality in Chinese institutional elderly persons. Journal of Clinical Epidemiology 50(10):1107-1116.

McCallum J, Shadbolt B, Wang D 1994. Self-rated health and survival: a seven-year follow-up study of Australian elderly. American Journal of Public Health 84 (7):1.100-1.105.

Montgomery MR, Gragnolati M, Burke K, Paredes E 1999. Measuring living standards with proxy variables. Policy Research Division Working Paper No. 129. The Population Council, Nova York. Disponível em $<$ www.popcouncil.org/pdfs/wp/129.pdf $>$.

Murray CJL, Chen LC 1992. Understanding morbidity change. Population and Development Review 18 (3): 481-503.

Ravaillon M 1994. Poverty comparisons. Harwood Academic Publishers, Chur, Suíça p. 10.

Sadana R, Mathers CD, Lopez AD, Murray CJL \& Iburg K 2000. Comparative analyses of more than 50 household surveys on health status. GPE Discussion Paper Series: No. 15. Disponível em <wwwnt.who.int/whosis/ statistics/discussion_papers/pdf/paper15.pdf $>$.

Sadana $\mathrm{R}$ et al.2002. Describing population health in six domains: comparable results from 66 household surveys. Organização Mundial da Saúde, GPE Discussion Paper No. 43. Disponível em <www3.who.int/ whosis/discussion_papers/pdf/paper43.pdf $>$.

Sen A 2002. Health: perception versus observation. Editorial British Medical Journal 324, 13 April:860-861.

Silva, PN 2002. Efeito do desenho amostral. Ciência e Saúde Coletiva 7(4).
Silveira MM 2002. Revelações do cotidiano sobre as definições de saúde e doença - uma experiência de ensino de epidemiologia. Revista Brasileira de Epidemiologia, Suplemento Especial139. Poster apresentado no V Congresso Brasileiro de Epidemiologia, Curitiba, 23 a 27 de março.

Sundquist J, Johansson SE 1997. Self reported poor health and low educational level predictors for mortality: A population based follow up study of 39.156 people in Sweden. Journal of Epidemiology and Community Health 51:35-40.

Tandon A, Murray JJL, Salomon JA, King G 2002. Statistical models for enhancing cross-country comparability. World Health Organization: Genebra, Suíça. GPE Discussion Paper no. 42. Disponível em < $<$ www3. who.int/whosis/discussion_papers/pdf/paper42.pdf $>$.

Valdivia M 2001. Acerca de la inequidad en salud en el Perú. Lima, Peru: Grupo de Análisis para el Desarrollo, Documento de trabajo. Disponível em $<$ www. grade.org.pe/eng/f-publicaciones.htm $>$.

van Doorslaer E, Jones AM 2002. Inequalities in self-reported health: validation of a new approach to measurement. University of York, Health Economics Resource Centre, Working Paper No.2. Disponível em $<$ www.york.ac.uk/res/herc/docs/WP2.pdf>.

Artigo apresentado em 8/8/2002

Aprovado em 31/8/2002

Versão final apresentada em 6/9/2002 